\title{
Direct read and quantify damage nucleotide from an oligonucleotide using a single molecule interface
}

\author{
Jiajun Wang ${ }^{1,2^{\ddagger}}$, Meng-Yin $\mathrm{Li}^{\ddagger}{ }^{\ddagger}$, Jie Yang ${ }^{\ddagger}$, Ya-Qian Wang ${ }^{2}$, Xue-Yuan Wu ${ }^{1,2}$, Jin Huang 3 , Yi-Lun Ying ${ }^{1}$, \\ Yi-Tao Long ${ }^{*}$ \\ ${ }^{1}$ State Key Laboratory of Analytical Chemistry for Life Science, School of Chemistry and Chemical Engineering, \\ Nanjing University, 210023, Nanjing, China. \\ ${ }^{2}$ School of Chemistry and Molecular Engineering, East China University of Science and Technology, 200237, Shanghai, \\ China \\ ${ }^{3}$ School of Pharmacy, East China University of Science and Technology, 200237, Shanghai, China. \\ Correspondence to Yi-Tao Long (yitaolong@nju.edu.cn). \\ ${ }^{\ddagger}$ These authors contribute equally.
}

KEYWORDS: Nanopore, Aerolysin, DNA, Electrochemistry, Single molecule interface

\begin{abstract}
DNA lesion such as metholcytosine $\left({ }^{\mathrm{m}} \mathrm{C}\right), 8$-OXO-guanine $\left({ }^{\circ} \mathrm{G}\right)$, inosine (I) etc could cause the genetic diseases. Identification of the varieties of lesion bases are usually beyond the capability of conventional DNA sequencing which is mainly designed to discriminate four bases only. Therefore, lesion detection remains challenge due to massive varieties and less distinguishable readouts for structural variations at molecular level. Moreover, standard amplification and labelling hardly works in DNA lesions detection. Herein, we designed a single molecule interface from the mutant K238Q Aerolysin, whose confined sensing region shows the high compatible to capture and then directly convert each base lesion into distinguishable current readouts. Compared with previous single molecule sensing interface, the resolution of the K238Q Aerolysin nanopore is enhanced by 2-order, which owns the best sensing performance in all reported aerolysin nanopores. In this work, the novel $\mathrm{K} 238 \mathrm{Q}$ could direct discriminate at least 3 types $\left({ }^{\mathrm{m}} \mathrm{C},{ }^{\circ} \mathrm{G}, \mathrm{I}\right)$ lesions without lableing and quantify modification sites under mixed hetero-composition condition of oligonucleotide. Such nanopore could be further applied to diagnose genetic diseases at high sensitivity.
\end{abstract}

Epigenetic modified DNA nucleotide causes protein dysfunction inducing tumor or even cancer diseases. Current, mass spectrometry and fluorometry are the approaches to identify the modified nucleotide. In a typical HPLC/mass spectrometry analysis, ${ }^{1}$ lesion units could be discriminate at high resolution, however requires tedious HPLC purification of the specific lesion part. Other reagent or labelling process using fluorometry provide feasibility as well as accuracy, but lack of generalization to each lesion variants. In addition, the lesion types, with the most common instance, methylated cytosine $\left({ }^{\mathrm{m}} \mathrm{C}\right)$ occurred at a frequency per 1 million repeats. ${ }^{2}$ Due to the massive types of lesion and the low occurrence, the sequencing and identification of the lesion unit in the heterogenous mixed condition is challenging for conventional approaches.

A generalized label-free single molecule technique is required to address these limitations. The nanopore technique can be the most prominent technique aiming DNA sequencing and lesion nucleotide detecting. In the proof-of-concept experiment, ${ }^{5}$ a single-stranded DNA molecule is driven through a -hemolysin under an electric field. Transient ionic current is obtained to direct measure the polynucleotide length. More recently, the nanopore catalyzed by phi29 DNA polymerase is engineered allowing real time detection of numerous nucleotide additions. ${ }^{3}$ Nanopore is also engineered by site directed mutagenesis to slow down the DNA translocation owing to stronger noncovalent interaction allowing base identification. ${ }^{4}$ Nonetheless, none of these techniques achieved the lesion detection in a mixed condition, owing to the mass contribution of alternated nucleotide base side chain is too small.

A more sensitive nanopore is required, for instance, MspA, ${ }^{5}$ ClyA, ${ }^{6}$ CsgG, ${ }^{7}$ Aerolysin, ${ }^{8}$ YaxAB complex, ${ }^{9}$ FraC, ${ }^{10}$ OmpG, ${ }^{11}$ Lysenin, ${ }^{12}$ CymA, $^{13}$ FhuA, ${ }^{14}$ etc. Alternatively, careful design of nucleotide chain form a hairpin structure or primer which could hook the segment inside the nanopore lumen. This approach allows the oxidative derivatives and even mispairing domain fall into the sensitive region to be detected. ${ }^{15-16}$ Among the above mentioned nanopores, Aerolysin from Aeromonas hydrophila owes the smallest pore diameter, $1.1 \mathrm{~nm}{ }^{17}$ The Wild-Type (WT) Aerolysin could identify single base difference ${ }^{8}$ and single methylated cytosine ${ }^{18}$ at the end of a 
homonucleotide mixture solution, nonetheless, the detection of multiple lesion types in a heteronucleotide mixture is still challenging.

In previous researches, ${ }^{19}$ it has been shown that the interaction between analyte and sensing interface of a nanopore as well as the steric effect both dominant the sensitivity. The confined space in a nanopore enhances the weak interaction force including the H-bond, van der Waal force, the electrostatic force so as to improve the single molecule probing capability. ${ }^{20}$ By testing with model nucleotide segment $\left(\mathrm{dA}_{4}\right)$, the residence time with prominent mutants such as K238E and K238G are 20 and 50 times longer than the WT, generating higher sensitivity. Nonetheless, during the statistical analysis process, the scatter distribution demonstrating the population of the two heteronucleotide oligonucleotides overlapped heavily suggesting the inefficient sensing ability of previous reported mutants (denoted as $\mathrm{S}$, correlates to the half-peak width following previous protocol ${ }^{28}$ ). Therefore, it is challenged for aerolysin nanopore to discriminate the mixture of more types of lesion units at different positions of a heteronucleotide. So far, a series of Aerolysin nanopore e.g. K238E, ${ }^{21} \mathrm{~K} 238 \mathrm{~F},{ }^{22} \mathrm{~K} 238 \mathrm{G},{ }^{22} \mathrm{R} 282 \mathrm{G},{ }^{23} \mathrm{Q} 212 \mathrm{R},{ }^{23} \mathrm{~N} 226 \mathrm{Q},{ }^{23}$
$\mathrm{K} 238 \mathrm{C},{ }^{24} \mathrm{~K} 238 \mathrm{Y}^{24} \mathrm{~K}^{238 \mathrm{C}^{25}}$ and other Aerolysin nanopores ${ }^{26-27}$ are designed, which attempt to the discrimination of single nucleotide in a heteronucleotide, at single molecule level. Nonetheless, none of the aforementioned mutants provides the sufficient sensing ability. Herein, we present the best aerolysin nanopore, K238Q, which shows the strongest sensing capability among all the reported biological nanopores in nucleotide detection. To consider the weak interaction between each base and 'key' amino acid (i.e. K238), Glutamine (Q) was then chosen due to its strong interaction with nucleotide bases. $^{29}$ Applying model nucleotide segment, $\mathrm{dA}_{4}$, the $\mathrm{K} 238 \mathrm{Q}$ reads the residue current and residence time as $34.5 \pm 0.5 \mathrm{pA}\left(\mathrm{I} / \mathrm{I}_{0}=0.58\right)$ and $122 \pm 19 \mathrm{~ms}$ respectively at 120 $\mathrm{mV}$, which induce a half-peak width $0.5 \mathrm{pA}$. Comparing to WT and all mutants, the time domain resolution has reached the best Aerolysin been so far reported, leading to an enhanced resolution of for single nucleotide discrimination by 2 -order. In this content, we demonstrate that the $\mathrm{K} 238 \mathrm{Q}$ nanopore could discriminate 3 types $\left({ }^{\mathrm{m}} \mathrm{C},{ }^{\circ} \mathrm{G}, \mathrm{I}\right)$ and quantify modifications under mixed hetero-composition condition.
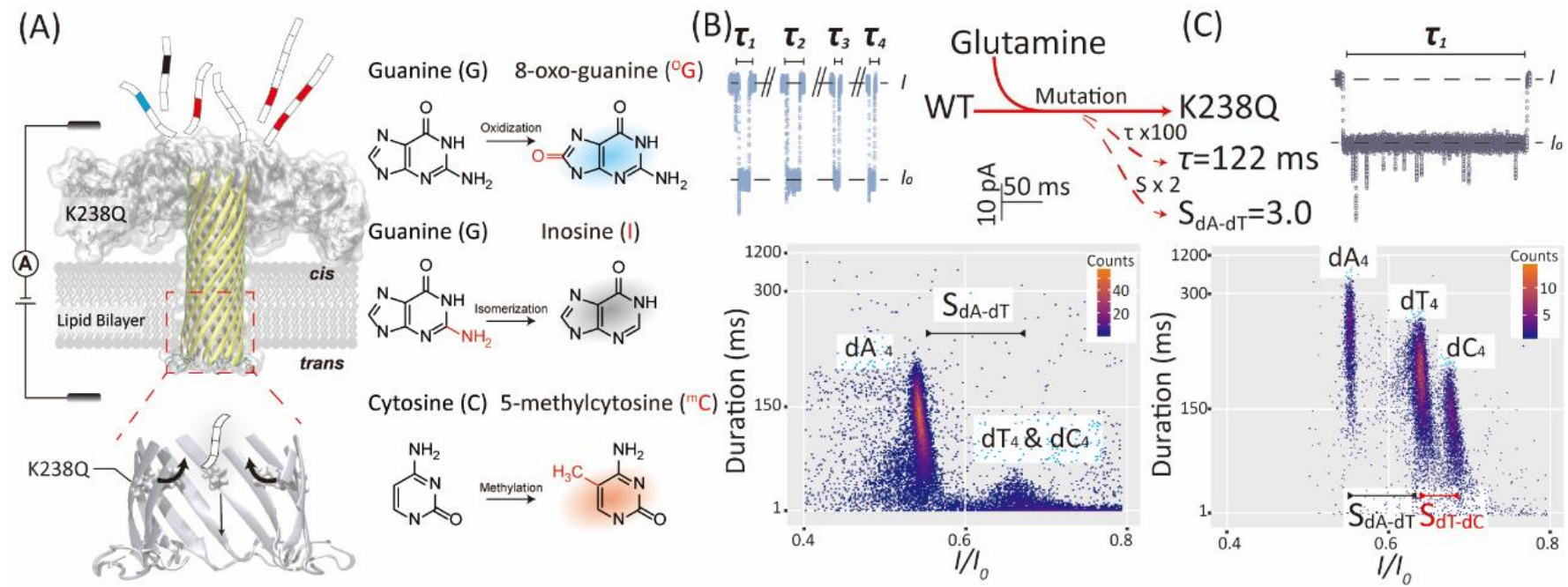

Figure $1 \mathrm{~K} 238 \mathrm{Q}$ Aerolysin nanopore measuring modified oligonucleotide. The modified nucleotide is 8-oxo-guanine, Inosine, 5-methylcytosine at different positions and quantification. (A) a typical K238Q Aerolysin inserted into artificial lipid bilayer with substrates added from cis side. Additional zoomed-in diagram showing the position of 238 site inside the Aerolysin lumen and the sensing mechanism to dA4. The implementation of Glutamine provides stronger interaction to model segment $\left(\mathrm{dA}_{4}\right)$ than WT. (B) Interaction signals from $\mathrm{dA}_{4}$ with $\mathrm{WT}$ aerolysin. The scatter plot shows the event distribution of $\mathrm{dX} 4 \mathrm{X}=\mathrm{A}, \mathrm{C}$, $\mathrm{T}), \mathrm{dT}_{4}$ and $\mathrm{dC}_{4}$ could not be discriminated. (C) Interaction signal from dA4 with $\mathrm{K} 238 \mathrm{Q}$. Residence time ( $\tau$ ) is prolonged 2 orders of magnitude, inducing better separation (S) capability. The scatter plot shows the event distribution of $\mathrm{dX}_{4}(\mathrm{X}=\mathrm{A}, \mathrm{C}, \mathrm{T})$, all can be discriminated.

\section{Results}

\section{Assessment of the new K238Q aerolysin nanopore}

We examined the sensing ability of K238Q with our model DNA, $\mathrm{dA}_{4}$. The residence time of the interaction is taken as an indicator which demonstrated 2 orders of magnitude increase than the WT, reached $122 \pm 19 \mathrm{~ms}$ at $120 \mathrm{mV}$ bias voltage. The prolonged residence time reflects that the $\mathrm{dA}_{4}$ and nanopore interaction is enhanced attributes to the glutamine (Q) replacement. The residue current (I) of the interaction is also regarded. In the case of $\mathrm{dA}_{4}$ probed by $\mathrm{K} 238 \mathrm{Q}$, the $\mathrm{I}_{\mathrm{res}}$ is ca. $34.5 \pm 0.5 \mathrm{pA}$ (corresponds to $\mathrm{I} / \mathrm{I}_{0}=0.58$ ) which is only slightly higher than WT $\left(\mathrm{I} / \mathrm{I}_{0}=0.51\right)$. Through the test with the model segment, the strong interaction between Glutamine (Q) and the nucleotide base reflects in the much longer residence time. The performance of K238Q discriminating $\mathrm{dA}_{4}$ with small half-peak width $(1.0 \mathrm{pA})$ suggesting good nucleotide identifying ability with great potential.

Then, other homo-oligonucleotide, deoxycytosine $\mathrm{dC}_{4}$, and deoxythymine $\mathrm{dT}_{4}$ were added successively to the $\mathrm{dA}_{4}$ measurement. Each substrate appeared distinguishable signature signal suggests the single K238Q aerolysin nanopore is capable to identify the $\mathrm{dX}_{4}(\mathrm{X}=\mathrm{A}, \mathrm{C}, \mathrm{T})$ from the mixed solution. The histogram of residue current show clear Gaussian distribution of the mixture of the $\mathrm{dX}_{4}$. Moreover, considering the peak distribution of $\mathrm{I}_{\text {res, }}$ the adenine contributes the smallest value $(34.5 \pm 0.5 \mathrm{pA})$, then cytosine $(40.1 \pm 1.0 \mathrm{pA})$ and thymine $(42.5 \pm 1.0 \mathrm{pA})$ The larger residue current corresponds to larger steric exclusion. Comparing to the molecular weight of the four 
deoxylnucleotide bases $(\mathrm{G}<\mathrm{A}<\mathrm{T}<\mathrm{C})$, the steric effect of single nanopore analysis however is in different ordering $(\mathrm{A}<\mathrm{G}<\mathrm{T}<\mathrm{C})$. The result suggests the non-covalent interaction is more pronounced than the steric effect interaction. From the structure inspection, the replacement of the positive charged arginine (K) with uncharged glutamine $(Q)$ makes the lumen more negatively charged due to the unpairing with the neighboring E258 causing decreased energy barrier for the cations. Moreover, the glutamine interact strongly with nucleotide base attributes to hydrogen bond as well as the van-der-Waals force..$^{29}$ Typically, the glutamine (Q) interacts strongest with Adenine, which in turn explained the best identification to our model segment, $\mathrm{dA}_{4}$. Moreover, $\mathrm{dA}_{4}, \mathrm{dC}_{4}$ and $\mathrm{dT}_{4}$ have a separation $(\mathrm{S})$ of $\mathrm{S}_{\mathrm{dA}-\mathrm{dT}}=3.67 \mathrm{pA}, \mathrm{S}_{\mathrm{dT}-\mathrm{dC}}=1.23 \mathrm{pA}$. The strongest non-covalent interaction between $\mathrm{Q}$ and $\mathrm{dA}$ provides the best separation ability of K238Q in discriminating the homo-nucleotide segments

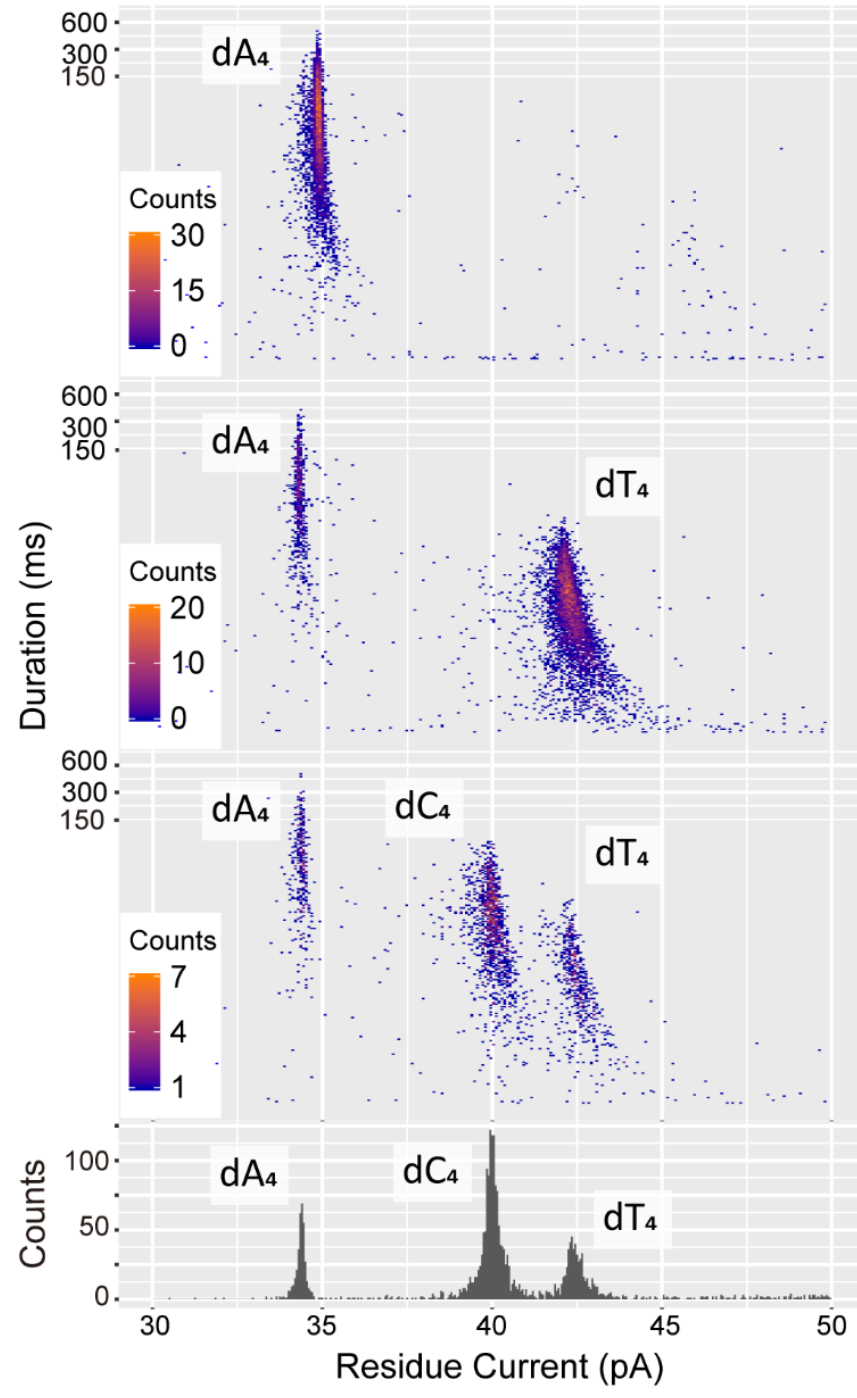

Figure 2 The distribution of current and duration from K238Q interaction with $\mathrm{X}_{4}(\mathrm{X}=\mathrm{A}, \mathrm{C}, \mathrm{T})$. The color bar illustrates the accumulation of each DNA segment. From the top figure, $\mathrm{A}_{4}$ was first introduced, then $\mathrm{C}_{4}$ and $\mathrm{T}_{4}$ were added successively with different peaks raised correlate to each segment. A histogram of the current distribution of the mixture was plotted at the bottom figure to demonstrate the current distribution and separation ability of K238Q. The data were acquired in $1.0 \mathrm{M} \mathrm{KCl}, 10 \mathrm{mM}$ Tris and 1.0 mM EDTA at pH 8.0 and $+120 \mathrm{mV}$ in the presence of $2.0 \mu \mathrm{M} \mathrm{dX} 4$.

\section{Single base detection}

WT aerolysin could discriminate homonucleotide segments, $5^{\prime}-\mathrm{XA}_{3}(\mathrm{X}=\mathrm{A}, \mathrm{G}, \mathrm{T}, \mathrm{C})$ in a mixture solution, ${ }^{30}$ while the discrimination of heteronucleotide remain poor attributes to the insufficient current resolution. To evaluate the sensitivity of $\mathrm{K} 238 \mathrm{Q}$ Aerolysin nanopore is capable to probe single nucleotide base in a heteronucleotide sequence, we then designed the heteronucleotide as $5^{\prime}$ XGTA (X=A, C, T, G). The heteronucleotide segments were mixed sequentially and examined by $\mathrm{K} 238 \mathrm{Q}$ and the results are given in Figure 3. The two current indicators, residue current and residence time of the blockages are examined. Regarding to residue current, the blockages show clear Ires which are centered at $44.40 \pm 0.22 \mathrm{pA}, 46.16 \pm 0.24 \mathrm{pA}$ and $46.78 \pm 0.40 \mathrm{pA}$ for AGTA, TGTA and CGTA respectively. The averaged residence time, AGTA contributes the largest duration which is $49.42 \mathrm{~ms}$, the TGTA and CGTA give the duration at $22.63 \mathrm{~ms}$ and $11.08 \mathrm{~ms}$ respectively. The current separation of the analytes are $\mathrm{S}_{\mathrm{AGTA} \text {-TGTA }}=1.76 \mathrm{pA}$, $\mathrm{S}_{\mathrm{TGTA} \text {-СGTA }}$ $=0.62 \mathrm{pA}$.

To this end, we have shown that the $\mathrm{K} 238 \mathrm{Q}$ could discriminate the single base difference in a heteronucleotide mixture without labelling or amplification process.

\section{Single base modification detection}

The WT aerolysin has showed their capability to discriminate the single methylation group at the $5^{\prime}$ end of a homonucleotide segments (i.e. CAAA vs. ${ }^{\mathrm{m}} \mathrm{CAAA}$ ) ${ }^{18}$. Later, mutant analog $\mathrm{K}^{2} 38 \mathrm{G}^{31}$ enables the discrimination of methylation occurred in the $2^{\text {nd }}$ position from $5^{\prime}$ end of a randomly designed heteronucleotide segments (i.e. ACGA $v s$. $\mathrm{A}^{\mathrm{m}} \mathrm{CGA}$ ). However, other types of epigenetic modification could not be properly discriminated due to the poor sensitivity. We herein show the ability of $\mathrm{K} 238 \mathrm{Q}$ in sensing the three types of DNA lesion (i.e. ${ }^{\circ} \mathrm{G}, \mathrm{I},{ }^{\mathrm{m}} \mathrm{C}$ ) occurred in a heteronucleotide segments.

The nucleotide, 5'-ACGA as the model segment is directly read by the K238Q. As examples of epigenetic modification, the guanine $(\mathrm{G})$ was modified into $I$ or ${ }^{\circ} \mathrm{G}$, respectively. Moreover, methylation was introduced into the cytosine (C). The modified model nucleotides are denoted as 5'-ACIA, 5'$\mathrm{AC}^{\circ} \mathrm{GA}, \quad 5^{\prime}-\mathrm{A}^{\mathrm{m}} \mathrm{CGA}$. As expected, the $\mathrm{K} 238 \mathrm{Q}$ could discriminate each type of lesion from each other as demonstrated in Figure 3(a). Following the mass increase order of 5'-ACIA, 5'-ACGA, 5'-A ${ }^{\mathrm{m} C G A}, 5^{\prime}-\mathrm{AC}^{\circ} \mathrm{GA}$, the histogram of the residue current centered at $59.32 \pm 0.44 \mathrm{pA}$, $58.61 \pm 0.42 \mathrm{pA}, \quad 57.28 \pm 0.35 \mathrm{pA}$ and $60.30 \pm 0.49 \mathrm{pA}$ respectively. The residence time are averaged at $11.37 \mathrm{~ms}$, $15.14 \mathrm{~ms}, 30.13 \mathrm{~ms}$ and $12.07 \mathrm{~ms}$. Neither the current nor the residence time matches the order of mass magnitude, moreover, the modification on the single nucleotide base makes ca. 20 Da difference in molecule weight, which weigh less than $2 \%$ of the model nucleotide. It is assumed that the separation of different types of epigenetic modification is achieved dominantly from the non-covalent interaction between nucleotide base and the sensing region rather than simply the steric effect which is negligible. Regarding to the separation capability of $\mathrm{K} 238 \mathrm{Q}$ in lesion detection, SAmCGA${ }_{A C G A}=1.33 \mathrm{pA}, S_{\text {ACGA-ACIA }}=0.71 \mathrm{pA}$ and $\mathrm{S}_{\text {ACIA-ACOGA }}=0.98 \mathrm{pA}$, 
which are much better than the K238G (data not shown). In order to prove the superior separation capability comes from the non-covalent interaction, the methylcytosine $\left({ }^{\mathrm{m}} \mathrm{C}\right)$ at different positions on a nucleotide segments is designed and examined by the K238Q.

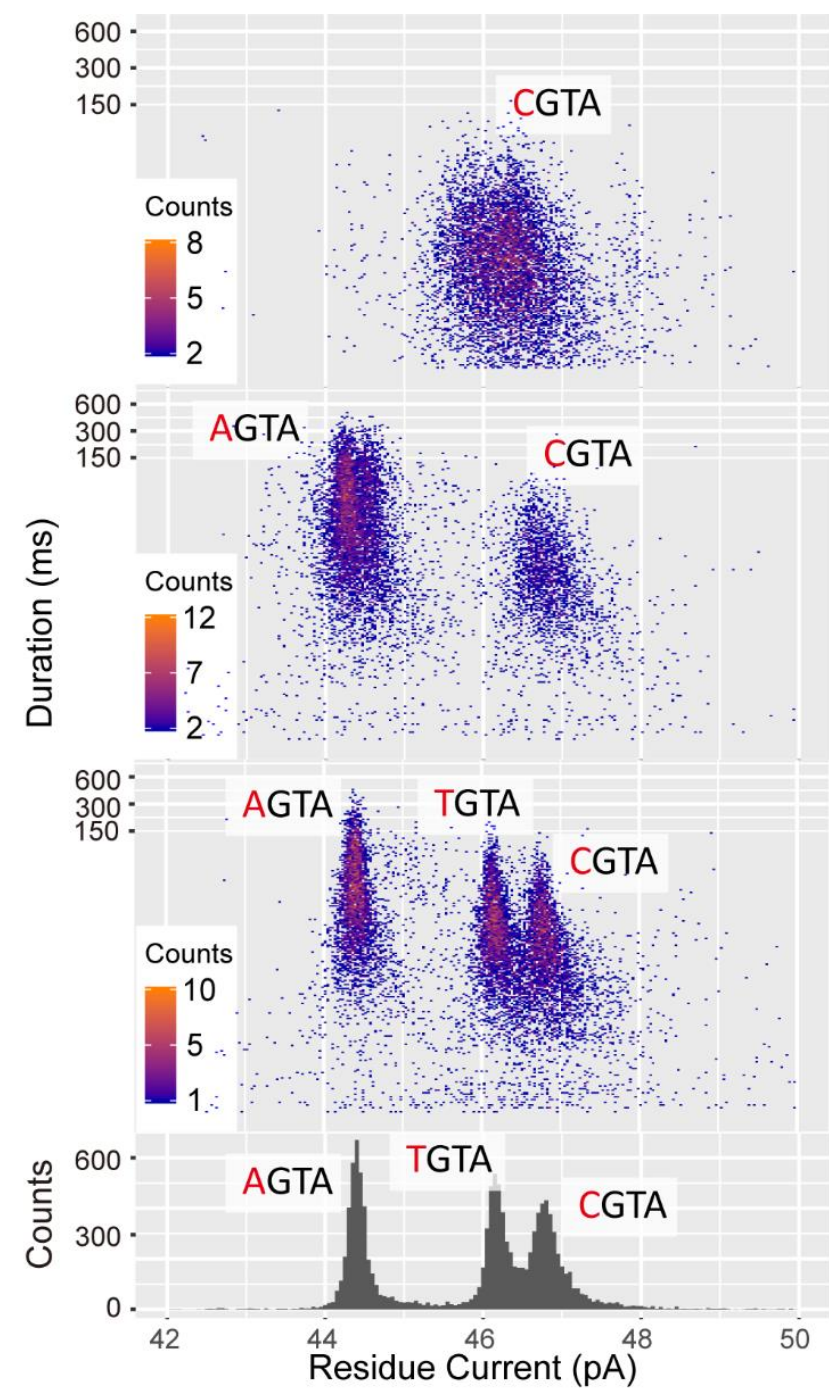

Figure 3 The distribution of current and duration from K238Q interaction with XGTA $(X=A, C, T)$. The color bar illustrates the accumulation of each DNA segment. From the top figure, AGTA was first introduced, then TGTA and CGTA were added successively with different peaks raised correlate to each segment. A histogram of the current distribution of the mixture was plotted at the bottom figure to demonstrate the current distribution and separation ability of K238Q. The data were acquired in $1.0 \mathrm{M} \mathrm{KCl}, 10$ $\mathrm{mM}$ Tris and $1.0 \mathrm{mM}$ EDTA at pH 8.0 and $+140 \mathrm{mV}$ in the presence of 2.0 $\mu \mathrm{M}$ XGTA.

Following the previous model segment, 5'-ACGA, another CG pair is introduced making a longer model nucleotide 5' ACGCGA. The cytosine at $2^{\text {nd }}$ and $4^{\text {th }}$ position are methylated. Since the weight of the methylation group is negligible and the nucleotide translocate linearly across the sensing region, the longer model nucleotide is modified into $5^{\prime}$ - $\mathrm{A}^{\mathrm{m}}$ CGCGA and $5^{\prime}-A^{m} C G^{m} C G A$. The mixture is probed by $\mathrm{K} 238 \mathrm{Q}$ and the residue current as well as residence time are adopted to analyze the blockages. The results are given in Figure 5 showing the powerful capability of K238Q in discriminating methylcytosine at different positions of a nucleotide segment. Such outcome could not be achieved by the other Aerolysin nanopores especially under label-free, heterocomposition and mixed condition.
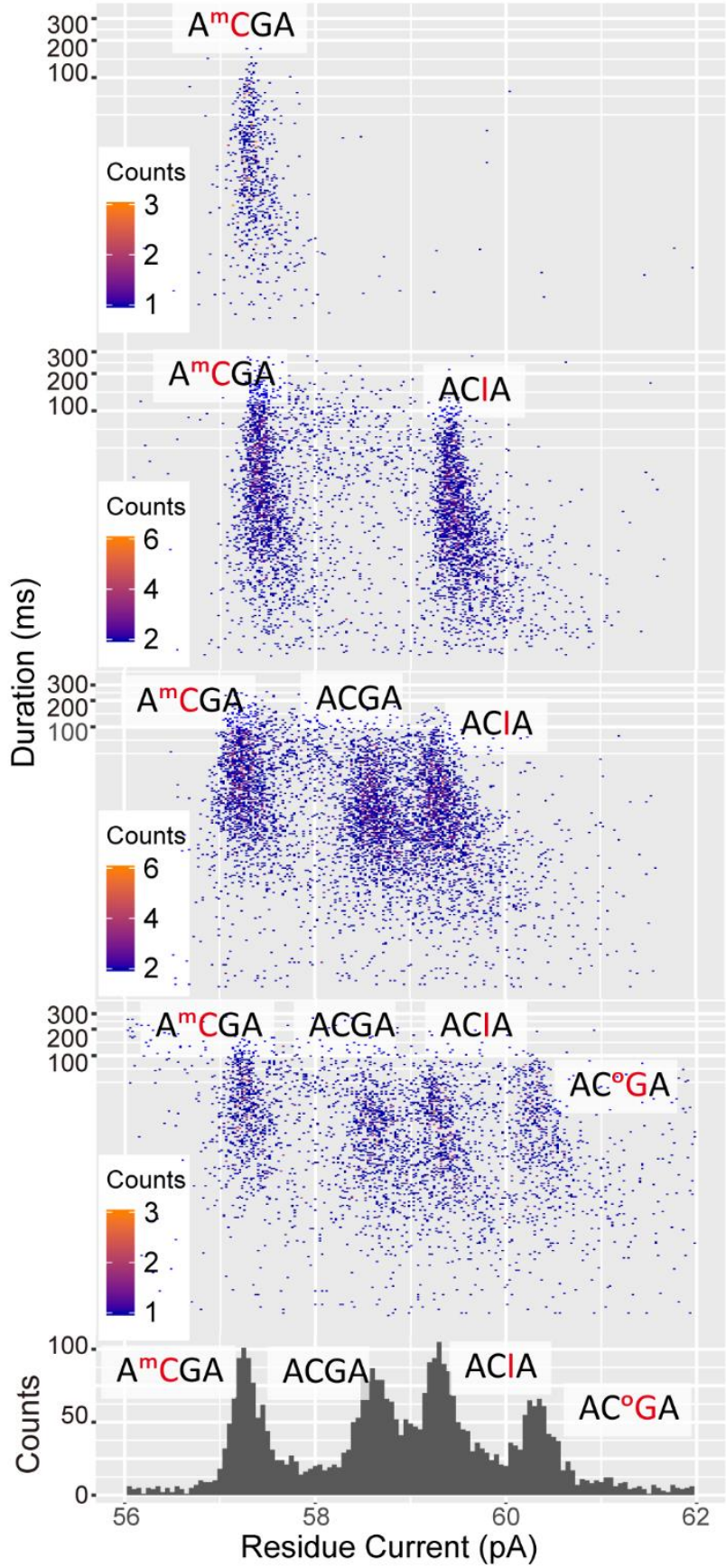

Figure 4 The distribution of current and duration from K238Q interaction with ACGA and the modified segments. The color bar illustrates the accumulation of each DNA segment. From the top figure, $\mathrm{A}^{\mathrm{m} C G A}$ was first introduced, then ACGA, ACIA and $\mathrm{AC}^{\circ} \mathrm{GA}$ were added successively with different peaks raised correlate to each segment. A histogram of the current distribution of the mixture was plotted at the bottom figure to demonstrate the current distribution and separation ability of K238Q. The data were acquired in $1.0 \mathrm{M} \mathrm{KCl}, 10 \mathrm{mM}$ Tris and $1.0 \mathrm{mM}$ EDTA at pH 8.0 and $+180 \mathrm{mV}$ in the presence of $2.0 \mu \mathrm{M}$ ACGA and the modified segments.

It is noteworthy that previous 5'-ACGA model showed mass contribution is negligible, the trend matches well with the non-covalent interaction from different position. More specifically, the outcome demonstrated the current distribution of detected ACGCGA, $\mathrm{A}^{\mathrm{m}}$ CGCGA and $\mathrm{A}^{\mathrm{m}} \mathrm{CG}^{\mathrm{m}} \mathrm{CGA}$ 
are centered at $36.36 \pm 0.58 \mathrm{pA}, 35.36 \pm 0.52 \mathrm{pA}$ and $33.42 \pm 0.49 \mathrm{pA}$ respectively. The residence time are averaged $39.7 \mathrm{~ms}, 26.1 \mathrm{~ms}$ and $21.8 \mathrm{~ms}$ respectively. Regarding to the separation ability of $\mathrm{K} 238 \mathrm{Q}$ to the modified base at different positions, $\mathrm{S}_{\text {AmCGmCGA-AmCGCGA }}=1.36$ $\mathrm{pA}, \mathrm{S}_{\text {AmCGCGA-ACGGGA }}=0.61 \mathrm{pA}$. Based on the previous oligonucleotides interaction with $\mathrm{K} 238 \mathrm{Q}$, the presence of Adenine interacts strongly with $Q$ which provides the longest residence time, while Cytosine interacts weakest with $\mathrm{Q}$ and provides relatively short residence time. Moreover, the nucleotide contains Adenine gives the largest separation than the other segments. According to this correlation, we could assume that the glutamine (Q) interacts stronger with methylcytosine $\left({ }^{\mathrm{m}} \mathrm{C}\right)$ than cytosine $(\mathrm{dC})$. Even ${ }^{\mathrm{m} C}$ position and quantification with glutamine (Q) could be measured or probed in the Aerolysin nanopore lumen with specified design.
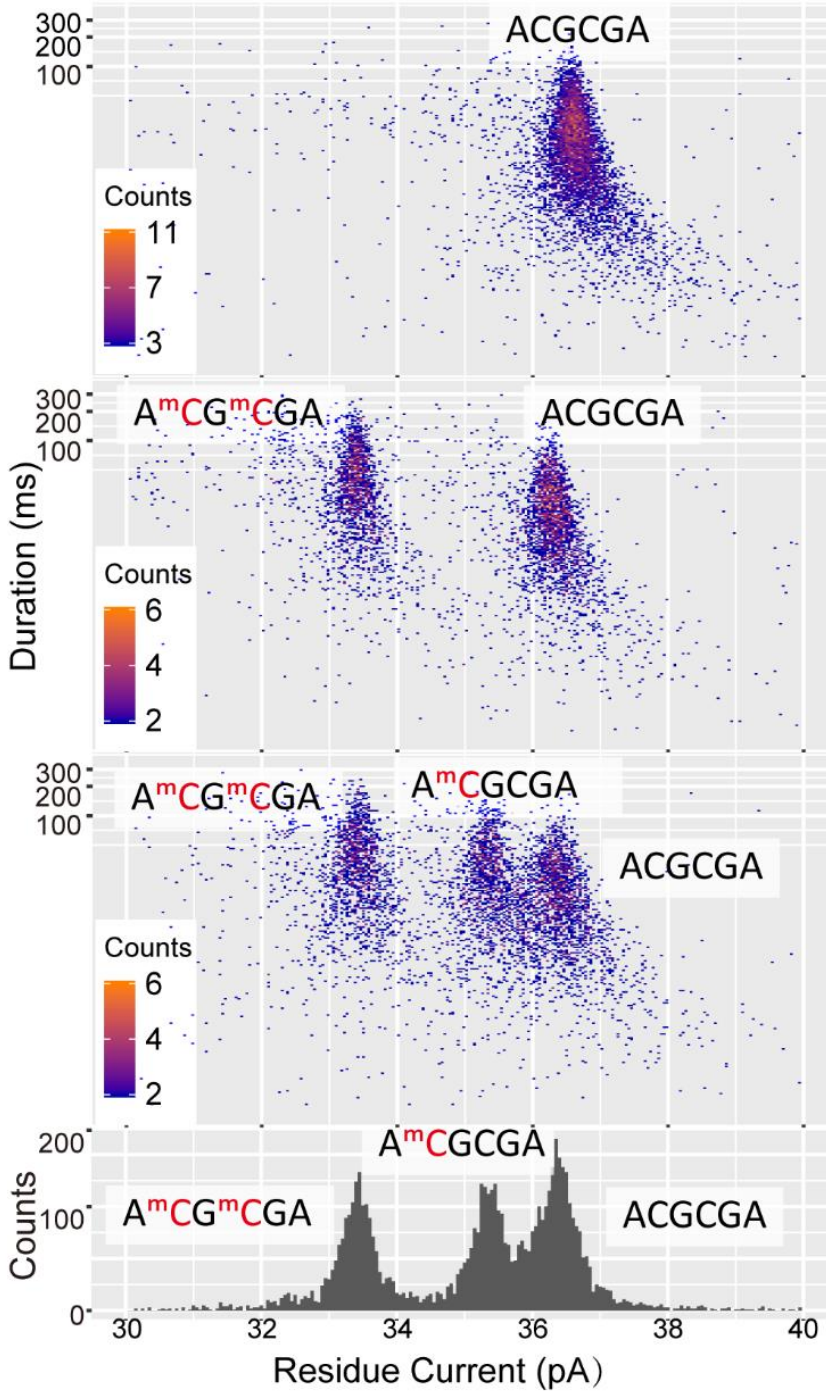

Figure 5 The distribution of current and duration from K238Q interaction with ACGCGA and the modified DNA segments. The color bar illustrates the accumulation of each DNA segment. From the top figure, $A^{\mathrm{m}} \mathrm{CG}^{\mathrm{m}} \mathrm{CGA}$ was first introduced, then $\mathrm{A}^{\mathrm{m}}$ CGCGA and ACGCGA were added successively with different peaks raised correlate to each segment. A histogram of the current distribution of the mixture was plotted at the bottom figure to demonstrate the current distribution and separation ability of K238Q. The data were acquired in $1.0 \mathrm{M} \mathrm{KCl}, 10 \mathrm{mM}$ Tris and $1.0 \mathrm{mM}$ EDTA at pH 8.0 and $+140 \mathrm{mV}$ in the presence of $2.0 \mu \mathrm{M}$ ACGCGA and the modified segments.

\section{Conclusion}

The K238Q Aerolysin nanopore achieved discrimination of different types of epigenetic modified nucleotide at different positions with no labelling and amplification. Point mutation $\mathrm{K} 238 \mathrm{Q}$ prolongs the nucleotide residence inside the nanopore lumen thus enhancing the non-covalent interaction to be distinguishable.

We first probed homo-nucleotide, taking example of $\mathrm{dA}_{4}$ the residence time is prolonged 2 orders of magnitude than WT. More importantly, neither of the two important parameters, residue current and residence time, showed correlation with the mass contribution, or steric effect. To prove this, we designed hetero-nucleotide with single base and even side chain difference. The K238Q well separated the mixture of these samples, again, not following the steric trend. Till this point, the epigenetic modifications contribute less than $2 \%$ of the weight, consequently, we assume the mass contribution is not dominant in our nanopore analysis and could be neglected. Then a longer model nucleotide with methylated cytosine at different positions are introduced for the purpose of single nucleotide base precise probing. Both the current and residence time decrease with the increase number of methylation at different positions. We finally could conclude that the non-covalent interaction is sensed and utilized for the separation of different types of epigenetic modifications at different positions.

Taking advantage of superior sensing ability to the epigenetic modification by $\mathrm{K} 238 \mathrm{Q}$, application could be applied to real-time monitoring the enzyme cleavage process $^{8}$ even achieving quantification different types of damaged nucleotide base. The analysis of longer nucleotide chain could be obtained by optimizing the measuring condition (e.g. $\mathrm{LiCl}, \mathrm{MgCl}_{2}$ ) ${ }^{32}$. Better current separation (S) could be achieved by applying an asymmetric salt concentration gradient. By controlling the amino acid composition at the sensing region, nucleotide bases and modified bases interaction with amino acid could be predicted, even the interaction between amino acid groups from a peptide could be mapped, due to its confined space. 33-36 Moreover, the parallelization and miniaturization of the nanopore based single molecule label-free detection method could be applied to real sample diagnose, with most prominent example such as the recognition of conformation change of homo-nucleotide with its application. ${ }^{37-38}$

\section{Materials and Methods}

All model nucleotides and modified nucleotides were synthesized and purified by Sangon Biotech Co., Ltd. (Shanghai, China). Chemical compounds such as the, $\mathrm{KCl}(\geqslant$ 99\%) and decane (anhydrous, $\geqslant 99 \%$ ) were purchased from Sigma-Aldrich Co., Ltd. (St. Louis, MO, USA). Phosphor lipid (1,2-diphytanoyl-snglycero- 3-phosphocholine) (powder, $\geqslant 99 \%$ ) was obtained from Avanti Polar Lipids, Inc. (Alabaster, AL, USA). The other chemical compounds were obtained from Aladine (Shanghai, China). Proaerolysin was obtained from Escherichia coli as previously described ${ }^{21,22}$. The Membrane formation and single nanopore experiments were performed identical to the standard protocol ${ }^{39}$. Single channel current was 
obtained from a patch clamp amplifier (Axon 200B) and digitized by an A/D converter (DigiData 1440A), Molecular Devices, Sunnyvale, CA, USA. All the data points were sampled at $100 \mathrm{kHz}$ and filtered at $5 \mathrm{kHz}$. Data analysis procedure follows the previous work ${ }^{30}$.

\section{ASSOCIATED CONTENT}

\section{Supporting Information.}

Fig S1 shows the I-V behavior of K238Q and WT Aerolysin. Fig S2 shows discrimination of XGTA by K238Q under asymmetry condition. Fig S3 shows discrimination of dG3 and dG4 by K238Q. Fig S4 shows discrimination of X3 by K238Q.

This material is available free of charge via the Internet at http://pubs.acs.org." For instructions on what should be included in the Supporting Information as well as how to prepare this material for publication, refer to the journal's Instructions for Authors.

\section{AUTHOR INFORMATION}

\section{Corresponding Author}

*Yi-Tao Long: yitaolong@nju.edu.cn

\section{Author Contributions}

Y-L. Y., Y-T. L. initiate the project. J. W., M-Y. L., J. Y. perform the experiments and data analysis. Y-Q. W., J. H. mutant the protein. J. W., M-Y. L., J. Y., Y-L. Y., Y-T. L. involve in the manuscript writing.

The manuscript was written through contributions of all authors.

†These authors contributed equally. (match statement to author names with a symbol)

\section{Funding Sources}

This research was supported by National Natural Science Foundation of China (21834001 and 61871183, 61901171). Yi-Lun Ying is sponsored by National Ten Thousand Talent Program for young top-notch talent. Jiajun Wang is sponsored by the Shanghai Sailing Program (19YF1410500), China Postdoctoral Science Foundation (2019M651412, 2019T120309).

\section{REFERENCES}

1. Cadet, J.; Poulsen, H., Measurement of oxidatively generated base damage in cellular DNA and urine. Free radical biology \& medicine 2010, 48 (11), 1457.

2. Wu, H.; Zhang, Y., Reversing DNA methylation: mechanisms, genomics, and biological functions. Cell 2014, 156 (1-2), 45.

3. Lieberman, K. R.; Cherf, G. M.; Doody, M. J.; Olasagasti, F.; Kolodji, Y.; Akeson, M., Processive replication of single DNA molecules in a nanopore catalyzed by phi29 DNA polymerase. Journal of the American Chemical Society 2010, 132 (50), 17961.

$4 . \quad$ Rincon-Restrepo, M.; Mikhailova, E.; Bayley, H.; Maglia, G., Controlled translocation of individual DNA molecules through protein nanopores with engineered molecular brakes. Nano Letters 2011, 11 (2), 746.

5. Butler, T. Z.; Pavlenok, M.; Derrington, I. M.; Niederweis, M.; Gundlach, J. H., Single-molecule DNA detection with an engineered MspA protein nanopore. Proceedings of the National Academy of Sciences 2008, 105 (52), 20647.

6. Soskine, M.; Biesemans, A.; Moeyaert, B.; Cheley, S.; Bayley, H.; Maglia, G., An engineered ClyA nanopore detects folded target proteins by selective external association and pore entry. Nano Letters 2012, 12 (9), 4895.

7. Goyal, P.; Krasteva, P. V.; Van Gerven, N.; Gubellini, F.; Van den Broeck, I.; Troupiotis-Tsaïlaki, A.; Jonckheere, W.; Péhau-Arnaudet, G.; Pinkner, J. S.; Chapman, M. R., Structural and mechanistic insights into the bacterial amyloid secretion channel CsgG. Nature 2014, 516 (7530), 250.

8. $\quad$ Cao, C.; Ying, Y.-L.; Hu, Z.-L.; Liao, D.-F.; Tian, H.; Long, Y.-T., Discrimination of oligonucleotides of different lengths with a wild-type aerolysin nanopore. Nature Nanotechnology 2016, 11 (8), 713.

9. Bräuning, B.; Bertosin, E.; Praetorius, F.; Ihling, C.; Schatt, A.; Adler, A.; Richter, K.; Sinz, A.; Dietz, H.; Groll, M., Structure and mechanism of the two-component $\alpha$ helical pore-forming toxin YaxAB. Nature communications 2018, 9 (1), 1806.

10. Huang, G.; Voet, A.; Maglia, G., FraC nanopores with adjustable diameter identify the mass of oppositecharge peptides with 44 dalton resolution. Nature communications 2019, 10 (1), 835.

11. Wang, J.; Bafna, J.; Bhamidimarri, S.-P.; Winterhalter, M., Small molecule permeation across membrane channels: Chemical modification to quantify transport across OmpF. Angewandte Chemie International Edition 2019, 58, 4737.

12. Podobnik, M.; Savory, P.; Rojko, N.; Kisovec, M.; Wood, N.; Hambley, R.; Pugh, J.; Wallace, E. J.; McNeill, L.; Bruce, M., Crystal structure of an invertebrate cytolysin pore reveals unique properties and mechanism of assembly. Nature communications 2016, 7, 11598.

13. van den Berg, B.; Bhamidimarri, S. P.; Prajapati, J. D.; Kleinekathöfer, U.; Winterhalter, M., Outermembrane translocation of bulky small molecules by passive diffusion. Proceedings of the National Academy of Sciences 2015, 112 (23), E2991.

14. Locher, K. P.; Rees, B.; Koebnik, R.; Mitschler, A.; Moulinier, L.; Rosenbusch, J. P.; Moras, D., Transmembrane signaling across the ligand-gated FhuA receptor: crystal structures of free and ferrichromebound states reveal allosteric changes. Cell 1998, 95 (6), 771.

15. Schibel, A. E. P.; An, N.; Jin, Q.; Fleming, A. M.; Burrows, C. J.; White, H. S., Nanopore Detection of 8-Oxo7,8-dihydro-2 '-deoxyguanosine in immobilized singlestranded DNA via adduct formation to the DNA damage site. Journal of the American Chemical Society 2010, 132 (51), 17992.

16. Jin, Q.; Fleming, A. M.; Johnson, R. P.; Ding, Y.; Burrows, C. J.; White, H. S., Base-excision repair activity 
of uracil-DNA glycosylase monitored using the latch zone of alpha-Hemolysin. Journal of the American Chemical Society 2013, 135 (51), 19347.

17. Iacovache, I.; De Carlo, S.; Cirauqui, N.; Dal Peraro, M.; van der Goot, F. G.; Zuber, B., Cryo-EM structure of aerolysin variants reveals a novel protein fold and the pore-formation process. Nature Communications 2016, 7, 12062.

18. Yu, J.; Cao, C.; Long, Y. T., Selective and Sensitive Detection of Methylcytosine by Aerolysin nanopore under serum condition. Analytical Chemistry 2017, 89 (21), 11685.

19. Cao, C.; Li, M.-Y.; Cirauqui, N.; Wang, Y.-Q.; Dal Peraro, M.; Tian, H.; Long, Y.-T., Mapping the sensing spots of aerolysin for single oligonucleotides analysis. Nature Communications 2018, 9 (1), 2823.

20. Ying, Y.-L.; Long, Y.-T., Nanopore-based singlebiomolecule interfaces: From information to knowledge. Journal of the American Chemical Society 2019, 141(40), 15720.

21. Wang, Y.; Cao, C.; Ying, Y.-L.; Li, S.; Wang, M.; Huang, J.; Long, Y.-T., Rationally designed sensing selectivity and sensitivity of an aerolysin nanopore via site-directed mutagenesis. ACS sensors 2018, 3(4), 779.

22. Wang, Y.-Q.; Li, M.-Y.; Qiu, H.; Cao, C.; Wang, M.B.; Wu, X.-Y.; Huang, J.; Ying, Y.-L.; Long, Y.-T., Identification of essential sensitive regions of the aerolysin nanopore for single oligonucleotide analysis. Analytical Chemistry 2018, 90 (13), 7790.

23. Wu, X.-Y.; Wang, M.-B.; Wang, Y.-Q.; Li, M.-Y.; Ying, Y.-L.; Huang, J.; Long, Y.-T., Precise construction and tuning of an aerolysin single-biomolecule interface for single molecule sensing. Chinese Chemical Society 2019, 1(3), 304.

24. Li, M.-Y.; Wang, Y.-Q.; Lu, Y.; Ying, Y.-L.; Long, Y., Single molecule study of hydrogen bond interactions between single oligonucleotide and aerolysin sensing interface. Frontiers in chemistry 2019, 7, 528.

25. Lu, Y.; Wu, X.-Y.; Ying, Y.-L.; Long, Y.-T., Simultaneous single-molecule discrimination of cysteine and homocysteine with a protein nanopore. Chemical Communications 2019, 55 (63), 9311.

26. Wang, J.; Li, M.-y.; Yang, J.; Wu, X.-y.; Huang, J.; Ying, Y.-l.; Long, Y.-t., Label-free detection of solo oligonucleotide lesion based on site-direct mutagenized aerolysin nanopore. Biophysical Journal 2019, 116 (3), 148 a.

27. Li, M.-Y.; Wang, Y.-Q.; Ying, Y.-L.; Long, Y.-T., Revealing the transient conformations of a single flavin adenine dinucleotide using an aerolysin nanopore. Chemical Science 2019, DOI: 10.1039/C9SC03163D.
28. Hu, Z. L.; Li, Z. Y.; Ying, Y. L.; Zhang, J.; Cao, C.; Long, Y. T.; Tian, H., Real-time and accurate identification of single oligonucleotide photoisomers via an aerolysin nanopore. Analytical Chemistry 2018, 90 (7), 4268.

29. Luscombe, N. M.; Laskowski, R. A.; Thornton, J. M., Amino acid-base interactions: a three-dimensional analysis of protein-DNA interactions at an atomic level. Nucleic acids research 2001, 29 (13), 2860.

30. Cao, C.; Yu, J.; Li, M.-Y.; Wang, Y.-Q.; Tian, H.; Long, Y.-T., Direct Readout of single nucleobase variations in an oligonucleotide. Small 2017, 13 (44), 1702011.

31. Wang, Y. Q.; Li, M. Y.; Qiu, H.; Cao, C.; Wang, M. B.; Wu, X. Y.; Huang, J.; Ying, Y. L.; Long, Y. T., Identification of essential sensitive regions of the aerolysin nanopore for single oligonucleotide analysis. Analytical Chemistry 2018, 90 (13), 7790.

32. Hu, Z.-L.; Li, M.-Y.; Liu, S.-C.; Ying, Y.-L.; Long, Y.$\mathrm{T}$., A lithium-ion-active aerolysin nanopore for effectively trapping long single-stranded DNA. Chemical Science 2019, 10 (2), 354.

33. Ying, Y.-L.; Zhang, J.; Gao, R.; Long, Y.-T., Nanopore-based sequencing and detection of nucleic acids. Angewandte Chemie-International Edition 2013, 52 (50), 13154.

34. Ying, Y.-L.; Cao, C.; Hu, Y.-X.; Long, Y.-T., A single biomolecule interface for advancing the sensitivity, selectivity and accuracy of sensors. National Science Review 2018, 5 (4), 450.

35. Wang, J.; Yang, J.; Ying, Y.-L.; Long, Y.-T., Nanopore-based confined spaces for single-molecular analysis. Chemistry - An Asian Journal 2019, 14(3), 389.

36. Zhen, G.; Yi-Lun, Y.; Yi-tao, L., Nanopore sensing system for high-throughput single molecular analysis. Science China Chemistry 2018, 6(12), 1483.

37. Guo, J.; Chen, Y.; Jiang, Y.; Ju, H., Polyadeninemodulated dna conformation monitored by surfaceenhanced raman scattering (SERS) on multibranched gold nanoparticles and its sensing application. Chemistry 2017, 23 (39), 9332.

38. Abbassi-Daloii, T.; Yousefi, S.; de Klerk, E.; Grossouw, L.; Riaz, M.; t Hoen, P. A. C.; Raz, V., An alanine expanded PABPN1 causes increased utilization of intronic polyadenylation sites. NPJ aging and mechanisms of disease 2017, 3, 6 .

39. Cao, C.; Liao, D.-F.; Yu, J.; Tian, H.; Long, Y.-T., Construction of an aerolysin nanopore in a lipid bilayer for single-oligonucleotide analysis. Nature Protocols 2017, 12 (9), 1901. 


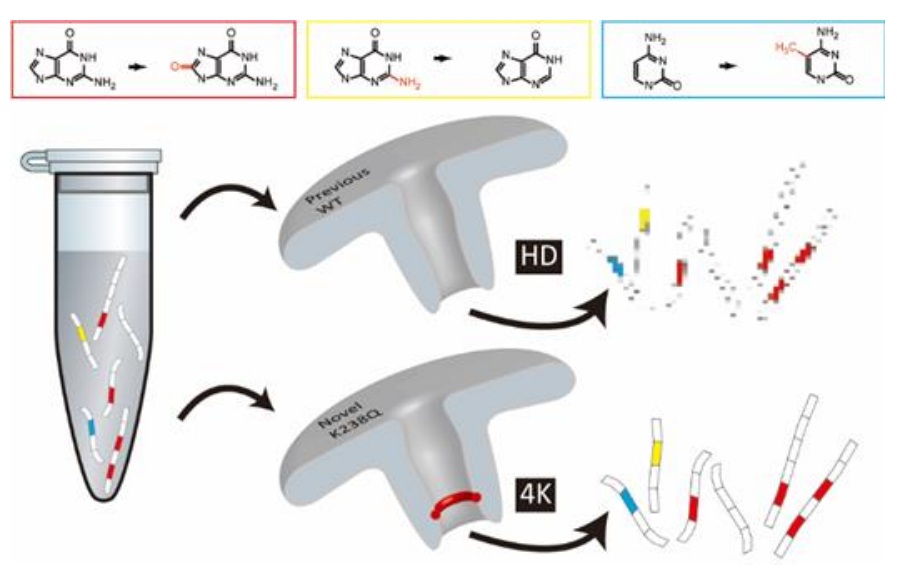

8 